\title{
Atenção farmacêutica ao idoso: investigação sobre conhecimento e aplicação prática
}

\author{
Pharmaceutical care for the elderly: an investigation on knowledge and practical \\ application
}

Samya Ribeiro Rezende ${ }^{1}$, Drielle Thainara Perez Paschoa ${ }^{2}$, Fernando Aucco Marim $^{3}$, Danila Fernanda Rodrigues Frias ${ }^{4}$

\section{RESUMO}

A atenção farmacêutica consiste na interação direta do profissional farmacêutico com o usuário, para que o paciente receba uma terapia eficiente e segura. $\mathrm{O}$ objetivo deste trabalho foi caracterizar uma população idosa com relação ao uso de fármacos e avaliar seu conhecimento sobre atenção farmacêutica. Foi desenvolvido um questionário com perguntas relacionadas a atenção farmacêutica e o uso racional de medicamentos. Dentre os entrevistados, $71,2 \%$ eram mulheres, $57,5 \%$ com faixa etária entre $60-69$ anos e $47,1 \%$ utilizavam anti-hipertensivos, $35,7 \%$ diuréticos e $24,3 \%$ antidepressivos. Dos participantes, $18,3 \%$ não sabiam qual a função dos medicamentos que ingeria e, afirmaram, $1,4 \%$ dos idosos, que faz uso de medicamento por indicação de parentes, e $11,3 \%$, já se confundiu no momento da ingestão do fármaco. Na pesquisa $47,5 \%$ disseram não saber o que é atenção farmacêutica. A maioria dos idosos utilizam medicamentos diariamente e não contam com auxílio farmacêutico para auxiliar no uso, o que pode causar problemas por isso, a atenção farmacêutica torna-se imprescindível a esta população.

Palavras-chave: Educação em Saúde. Geriatria. Saúde Pública. Tratamento Farmacológico.

\section{ABSTRACT}

Pharmaceutical care consists of the direct interaction of the pharmaceutical professional with the user, so that the patient receives an efficient and safe therapy. This work seeks to characterize an elderly population regarding the use of drugs and evaluate their knowledge about pharmaceutical care. A questionnaire was developed with questions pertaining to pharmaceutical care and the rational use of medicines. Among the interviewees, $71.2 \%$ were women, $57.5 \%$ were aged $60-69$ years and $47.1 \%$ used antihypertensive drugs, $35.7 \%$ diuretics and $24.3 \%$ antidepressants. Of all participants, $18.3 \%$ did not know the function of the drug(s) they were taking, and according to the results, $1.4 \%$ of the elderly use medication as indicated by relatives and $11.3 \%$ of these reporting having already been confused at the time of ingestion the drugs. In the survey $47.5 \%$ stated they did not know what pharmaceutical care is. The majority of the elderly use medication daily and do not count on pharmaceutical assistance to obtain assistance in its use, which can cause problems. Therefore, pharmaceutical attention is indispensable for this population.

Keywords: Drug Therapy. Geriatrics. Health Education. Public health.
${ }^{1}$ Discente do curso de farmácia, Universidade Brasil, Campus Fernandópolis.

E-mail:

samyarezendelcg@gmail.com

2 Discente do curso de farmácia, Universidade Brasil, Campus Fernandópolis.

E-mail:

drielle.paschoa@hotmail.com

${ }^{3}$ Discente do curso de farmácia, Universidade Brasil, Campus Fernandópolis.

E-mail: fermarim97@gmail.com

${ }^{4}$ Docente do Programa de PósGraduação Stricto Sensu em Ciências Ambientais, Universidade Brasil, Campus Fernandópolis.

E-mail: danila.frias@ub.edu.br 


\section{INTRODUÇÃO}

A atenção farmacêutica consiste em uma estratégia de atuação social e multidisciplinar do farmacêutico junto a sociedade. Esta estratégia é muito importante pois visa a diminuição da morbimortalidade principalmente de idosos relacionada ao uso de medicamentos (MENESES; SÁ, 2010; SILVA, NASCIMENTO, GRASSI, 2016).

Esta prática deve estar sempre voltada a orientação e acompanhamento farmacêutico, a educação em saúde, a dispensa do uso de medicamentos, e ao registro sistemático de atividades com mensuração e avaliação dos resultados (MENESES, SÁ, 2010).

A atenção farmacêutica é a interação direta do profissional farmacêutico com o usuário, cujo objetivo é que o paciente receba uma terapia eficiente e segura para melhoria de sua qualidade de vida (IVAMA et al., 2002).

Estima-se que em 2025, a população brasileira acima de 60 anos tenha aumentado 15 vezes com relação a existente em 1950, alcançando a marca de mais de 35 milhões de idosos, e que o Brasil assumirá a sexta posição de país mais envelhecido do mundo (NOBREGA, KARNIKOWSKI, 2005; SILVA, NASCIMENTO, GRASSI, 2016). Este fato pode estar relacionado à queda da taxa de fecundidade e aumento do índice de expectativa de vida no Brasil (ROMANO-LIEBER et al., 2002; NOVAES, 2007).

Os idosos são considerados os grandes consumidores de medicamentos devido a ocorrência de problemas de saúde crônicos nesta idade ser maior. Além disso, constituem cerca de $50 \%$ dos multiusuários, ou seja, usam mais de um medicamento ao mesmo tempo (ANDRADE, SILVA, FREITAS, 2004; BURTON et al., 2005; LOYOLA FILHO et al., 2006; AGUIAR et al., 2008).

Desta forma, os idosos acabam sendo mais expostos a erros em suas prescrições, como dosagens, indicações inadequadas, interações medicamentosas, associações com redundância, o que os torna mais susceptíveis a ocorrência de reações adversas (ANDRADE, SILVA, FREITAS, 2004).

Outro fator importante com relação aos idosos é o fato da idade avançada contribuir para erro de autoadministrações medicamentosas por meio da confusão causada pela quantidade excessiva de medicamentos diários, a visão prejudicada que faz confundir as caixas dos fármacos semelhantes, destreza manual prejudicada e distúrbios cognitivos (LUPPI, CARVALHO, 2005). 
Cerca de $90 \%$ dos idosos do Brasil consomem pelo menos um tipo de medicamento diariamente, e destes um terço consomem de cinco a mais fármacos simultaneamente, caracterizando a chamada polifarmácia, e estes medicamentos são prescritos ou vendidos livremente (SILVA, NASCIMENTO, GRASSI, 2016).

Um fator que contribui significativamente com a prática da polifarmácia pelos idosos é a variedade e disponibilidade de fármacos em supermercados, drogarias e farmácias, geralmente alvos de promoções e descontos, o que estimula o consumo, prática esta danosa, pois estimulando o uso de medicamentos aumenta-se a possibilidade de intoxicações e reações adversas do usuário (ANDRADE, SILVA, FREITAS, 2004).

A atenção farmacêutica ao idoso é essencial, pois auxilia e os incentiva a efetuar o uso correto dos medicamentos. Assim, a intervenção farmacêutica por meio de ações educativas e de orientações promove benefícios a saúde do paciente. Porém para que esta prática seja realizada de forma correta, a mesma deverá ser executada por um profissional capacitado, com empenho e responsabilidade, adquiridos na formação acadêmica e na vivência profissional (MENESES, SÁ, 2010).

Infelizmente a prática de atenção farmacêutica ao idoso é pouco realizada, o que dificulta a adoção de medidas que previnam os agravos a saúde dos idoso causadas pelo uso irracional de medicamentos (MENESES, SÁ, 2010). Por isso, o objetivo nesta pesquisa foi caracterizar uma população idosa frequentadora de um Centro de Convivência de Idosos com relação ao uso de fármacos e avaliar seu conhecimento sobre atenção farmacêutica.

\section{MATERIAL E METODOS}

A pesquisa foi desenvolvida no Município de Meridiano, São Paulo, junto ao Centro de Convivência do Idoso (CCl) da cidade, após autorização do responsável local.

Foi desenvolvido um questionário estruturado com questões relacionadas a atenção farmacêutica e o uso racional de medicamentos. O mesmo foi aplicado, por meio de entrevistas, a 80 idosos cadastrados e que frequentavam o Centro de Convivência do Idoso de Meridiano, São Paulo, durante o período de outubro de 2019 e janeiro de 2020. Os entrevistados foram selecionados de forma aleatória dentro da população do estudo, compreendida por 100 idosos.

A participação dos indivíduos realizou-se mediante a assinatura do termo de consentimento livre e esclarecido (TCLE), sendo assegurado aos participantes sigilo quanto às informações prestadas e todo esclarecimento necessário sobre o estudo. A pesquisa foi aprovada pelo comitê de ética da Universidade Brasil com parecer número 2.648.183. 
Após realização das entrevistas, os dados obtidos foram digitalizados e tabulados em planilhas do software Microsoft Office Excel formando o banco de dados. Em seguida, os dados foram analisados por meio de estatística descritiva (cálculo de médias simples e porcentagem). Os resultados foram apresentados no formato de tabelas e gráficos.

\section{RESULTADOS E DISCUSSAO}

O estudo foi realizado com 80 idosos que frequentavam o $\mathrm{CCl}$ de Meridiano, SP. O perfil dos entrevistados está descrito na Tabela 1.

Tabela 1. Perfil dos idosos entrevistados no CCI de Meridiano, São Paulo, 2019

\begin{tabular}{c|c|c|c}
\hline SEXO & ESTADO CIVIL & ESCOLARIDADE & \multicolumn{1}{c}{ FAIXA ETÁRIA } \\
\hline Feminino (71,2\%) - 57 & Solteiro (10\%)-8 & Educação Básica (18,7\%) - 15 & $60-69(57,5 \%)-46$ \\
Masculino (28,8\%)-23 & Casado (58,8\%)-47 & Ensino Fundamental (41,3\%) - 33 & $70-79(33,7 \%)-27$ \\
& Divorciado (7,5\%)-6 & Ensino Médio (30\%) - 24 & $80-89(6,3 \%)-5$ \\
& Viúvo (23,7\%)-19 & Ensino Superior (10\%) - 8 & $90-99(2,5 \%)-2$ \\
\hline
\end{tabular}

Fonte: Elaborada pelos autores

O predomínio de mulheres em $\mathrm{CCl}$ já foi descrito em outras pesquisas, assim como neste estudo (BATISTA, VIEIRA, SILVA; 2012; SOUZA NETO, et al., 2018; SILVA, 2019). Este dado pode estar relacionado ao fato de as mulheres procurarem mais o Centro de Convivência do Idoso ( $\mathrm{CCl}$ ) em busca de novas amizades e companhia, onde encontram pessoas da mesma faixa etária e podem trocar experiências. Além disso, este achado confirma a ocorrência do processo chamado "feminilização da velhice", que ocorre no mundo todo e está relacionado a população idosa ser composta por mulheres em sua maioria, além da maior mortalidade masculina (NICODEMO, 2010; SILVA, 2019).

Neste estudo, 57,5\% dos idosos possuíam entre 60-69 anos. Pesquisas realizadas em Terezina, Pl, corroboraram com o estudo com relação a idade dos frequentadores do CCI (BATISTA, VIEIRA, SILVA, 2012; FREIRE et al., 2015). Já Silva (2019) e Souza Neto et al. (2018), referiram que os idosos que mais frequentam CCI são da faixa etária entre 70 a 80 anos, considerados medianamente idosos, diferente desta pesquisa, onde sobressaiuse a faixa etária dos chamados idosos jovens. Este fato pode indicar que os idosos estão procurando cada vez mais cedo obter novas amizades e companhias por meio da presença em locais especializados em acompanhamento de idosos.

A escolaridade dos idosos entrevistados, caracterizou-se pela maioria $(41,3 \%)$, o ensino fundamental. Batista, Vieira, Silva (2012) e Souza Neto et al. (2018) também detectaram em seus estudos que a maioria dos idosos possuíam ensino fundamental. A 
baixa escolaridade entre os idosos confirma estudos de base populacional (BATISTA, VIEIRA, SILVA, 2012). Mas também pode estar relacionada ao tipo de atividade desenvolvida nestes locais, pois não exigem escolaridade para que sejam executadas, ou seja, a participação e o entendimento das ações são facilmente exequíveis por todos.

O estado civil que mais se destacou na pesquisa foi o casado (58,8\%), seguido pelo viúvo $(23,7 \%)$. Alguns estudos encontraram resultados semelhantes ao desta pesquisa, pois Souza Neto et al. (2018), destacaram que 35\% dos idosos entrevistados eram casados e $27 \%$ viúvos, assim como Freire et al. (2015), que citaram $36,2 \%$ de casados e $30,7 \%$ de viúvos.

Ainda, foi averiguado neste estudo, que a maioria dos idosos que citaram estado civil viúvo eram do sexo feminino, e os casados, do sexo masculino. Estes dados são semelhantes aos citados por Batista, Vieira, Silva (2012). Esta informação pode estar relacionada ao fato das mulheres, após ficarem viúvas, ter a tendência de manter-se sozinhas, enquanto os homens geralmente se casam novamente. Saldanha, Caldas (2004) afirmaram que o número de viúvas é superior ao de viúvos, no mundo todo.

Dentre os entrevistados, $88,8 \%$ utilizam medicamentos de uso continuo. Freitas; Toma (2019), também citaram o consumo de medicamentos contínuos por $75 \%$ dos idosos entrevistados, e Souza Neto et al. (2018), por $87,8 \%$.

As doenças afetam mais a população idosa, desta forma os medicamentos são importantes, porém perigosos. Importantes pois auxiliam no prolongamento e melhoria de qualidade de vida, e perigosos, pois o consumo de forma irracional e as dificuldades que os idosos encontram no momento de consumir o fármaco pode o expor a risco iminentes (MARIN et al., 2008).

Devido ao aumento de patologias que ocorrem com o passar da idade, a elevação do consumo de medicamentos é inevitável. Desta forma, estes pacientes ficam mais propensos a cometer erros de administração, principalmente devido ao número elevado de medicamentos, associados a idade avançada, que traz consigo os distúrbios cognitivos, a dificuldade visual e a perca de destreza manual (SILVA, NASCIMENTO, GRASSI, 2016).

Dentre os idosos que faziam uso de medicamentos contínuos, os relatos referentes as indicações estão descritos na Figura 1. 


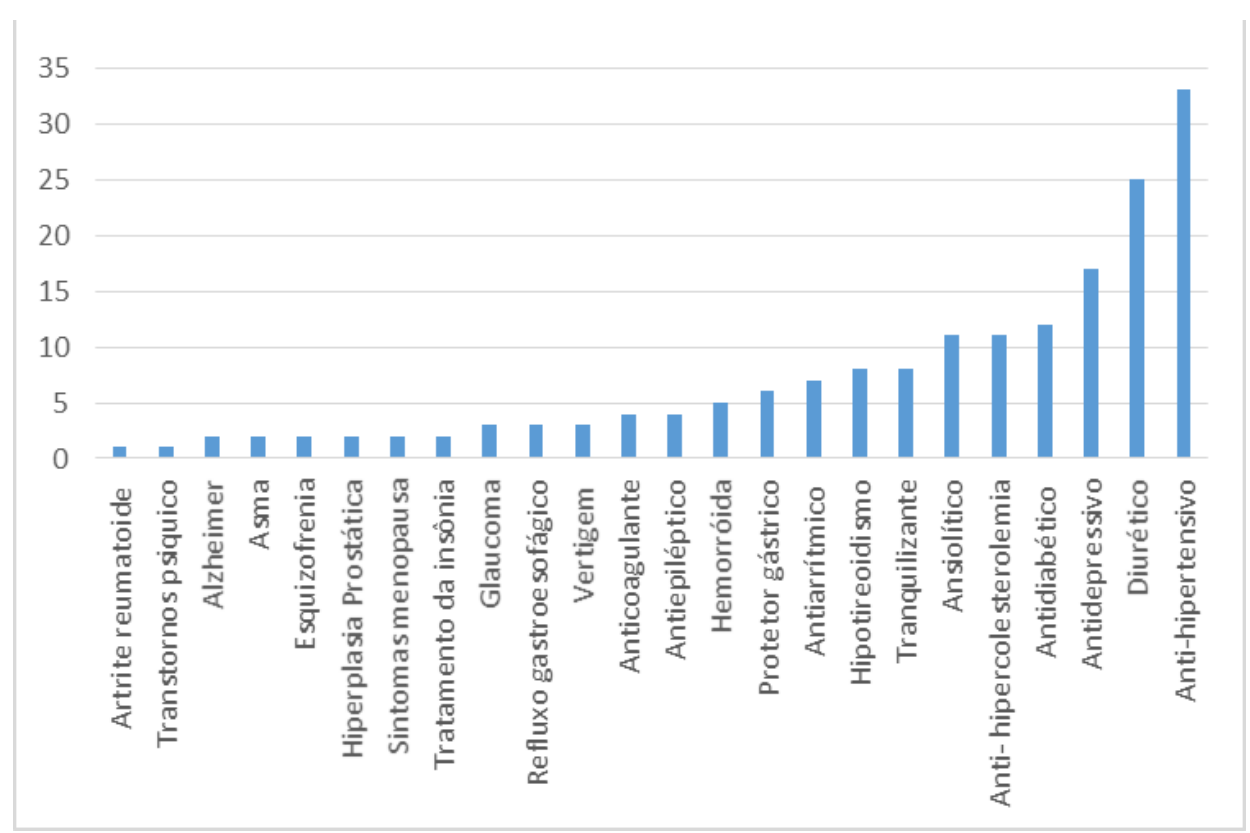

Figura 1. Indicações dos medicamentos consumidos diariamente por idosos entrevistados em Meridiano, São Paulo, 2020

Fonte: Elaborada pelos autores

Notou-se que a maioria (47,1\%) fazem uso de anti-hipertensivos, seguido de $35,7 \%$ que consomem diuréticos, 24,3\% antidepressivos, 17,2\% antidiabéticos, 15,7\% antihipercolesterolemicos, e 15,7\% ansiolíticos. Doenças crônicas como a hipertensão e a diabetes são comuns em idosos, por isso estes indivíduos acabam por ser os maiores consumidores de medicamentos de uso contínuo, sendo a faixa etária considerada mais medicada dentre a população (COSTA; ALVES; JESUS, 2019).

A hipertensão é a doença principal que afeta indivíduos adultos acima de 60 anos (FREIRE et al., 2015; MALAQUIAS et al., 2016; FREITAS; TOMA, 2019; SILVA, 2019). Outras doenças, assim como as detectadas neste estudo, também foram prevalentes em idosos avaliados em outras pesquisas, como a hipercolesterolemia, diabetes, depressão e doenças cardíacas (FREIRE et al., 2015; SOUZA NETO et al., 2018; FREITAS; TOMA, 2019; PARRINI; PINHEIRO; SILVA, 2019).

Os medicamentos mais utilizados para controle das afecções citadas pelos idosos na Figura 1, estão descritos na Figura 2. 


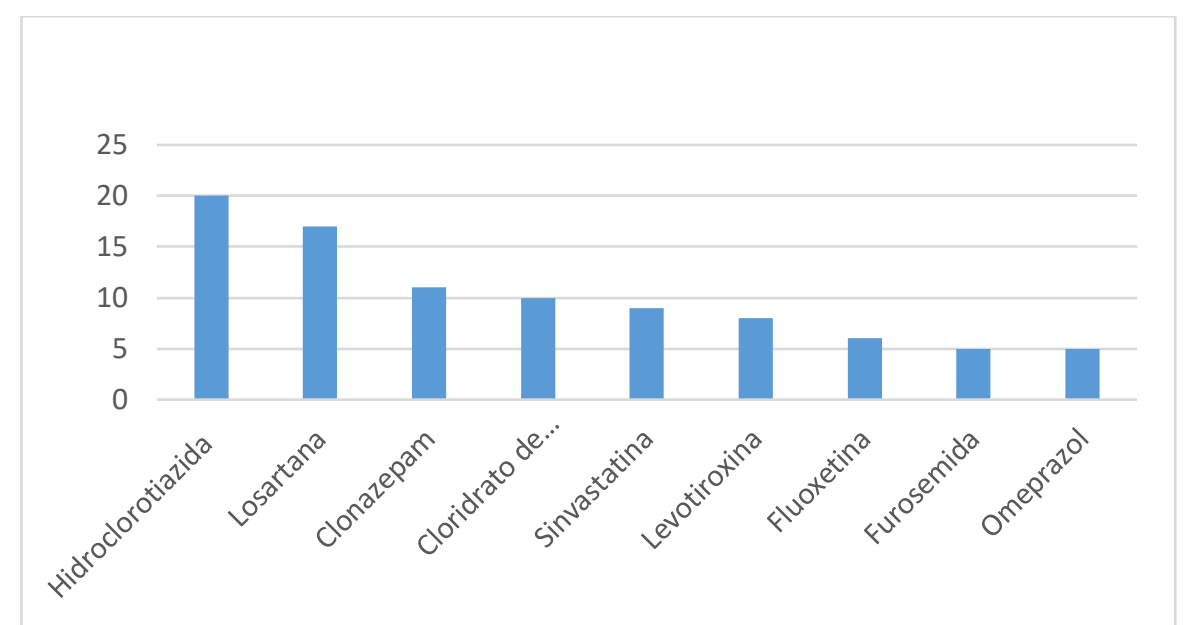

Figura 2. Principais princípios ativos utilizados diariamente pelos idosos entrevistados em Meridiano, São Paulo, 2020

Fonte: Elaborada pelos autores

A hidroclorotiazida e a losartana são os princípios ativos mais utilizados. Este fato está relacionado a maioria dos idosos sofrerem com hipertensão, sendo estes fármacos indicados para o tratamento desta enfermidade.

A losartana é um dos medicamentos mais vendidos no Brasil para tratamento da pressão arterial elevada e insuficiência cardíaca. Já a hidroclorotiazida é um diurético que pode ser utilizado em associação a outro fármaco, inclusive a losartana, para tratamento de pressão arterial elevada (CONCEIÇÃO et al., 2019).

Freitas; Toma (2019), relataram em sua pesquisa que os medicamentos de uso continuo mais utilizados pelos idosos eram os relacionados ao tratamento da hipertensão arterial e de hipercolesterolemia. Já Parrini; Pinheiro; Silva (2019), citaram o uso principalmente de anti-hipertensivos, diuréticos, antidepressivos, antiulcerosos e hipolipemiantes. Todas estas informações coincidem com as encontradas neste trabalho.

Devido ao elevado uso de medicamentos controlados, principalmente os antidepressivos e ansiolíticos, a atenção farmacêutica ao paciente idoso deve ser redobrada. Estes medicamentos, quando indicados para uso em indivíduos da terceira idade tornam-se muito mais perigosos pois os riscos de erros de ingestão causados por falhas provocadas pela idade avançada aumentam significativamente (CAZARIM; ARAUJO, 2012).

Com relação ao número de medicamentos de uso contínuo consumidos diariamente pelos idosos, os resultados estão expressos na Figura 3. 


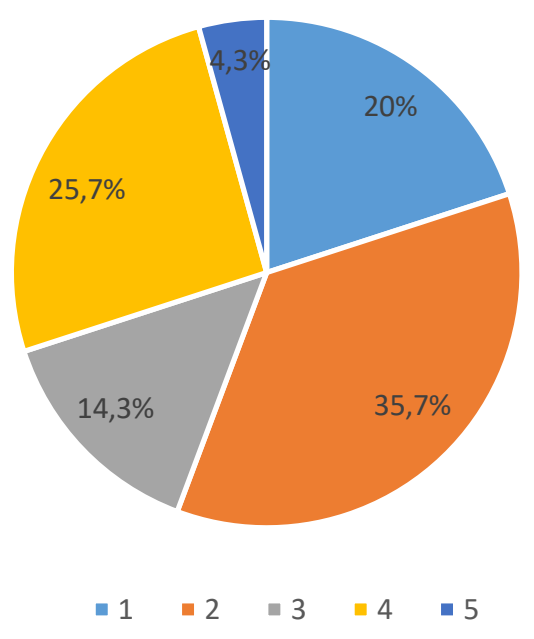

Figura 3. Quantidade de medicamentos consumidos de uso continuo diariamente por idosos entrevistados em Meridiano, São Paulo, 2020

Fonte: Elaborada pelos autores

Destacou-se na pesquisa o uso de $2(35,7 \%)$ e $4(25,7 \%)$ medicamentos de uso continuo diariamente pelos idosos entrevistados. Silva; Nascimento; Grassi (2016) afirmaram que $90 \%$ dos idosos consomem ao menos um medicamento ao dia, porém, muitos idosos consomem mais de quatro, sendo eles fármacos prescritos ou não.

Devido a elevada ocorrência de patologias em pessoas idosas, principalmente as crônicas, e o aumento da expectativa de vida, é comum a prática chamada polifarmácia, ou seja, uso de múltiplos medicamentos. Esta prática impacta diretamente na qualidade de vida dessas pessoas, pois induz ao erro de administração e consequentemente aos riscos de ocorrência de reações adversas (MANSO et al., 2015; CADOGAN et al., 2016).

A atuação do farmacêutico nestes casos é fundamental, pois trata-se de um profissional que também é responsável pelo paciente. Ele deve alertar ao idoso as possíveis reações adversas, o risco de intoxicações, as prováveis interações, fazendo com que o consumo do medicamento seja realizado de forma correta e eficaz (LIMA et al., 2016; COSTA; ALVES; JESUS, 2019).

Cuidados na prescrição também devem ser tomados pelos médicos. Duplicidade de terapêutica por mecanismo de ação, subdosagem de medicamentos, iatrogenia entre associações de antidepressivos, já foram relatados em estudos e provocam sérios danos à saúde do paciente (SCHRODER et al., 2019). Por isso, os profissionais da saúde devem sempre adotar medidas profiláticas relacionadas ao consumo inadequado de fármacos. 
Desta forma, o uso de receitas legíveis, contendo prescrições adequadas com o menor número possível de fármacos e a menor dosagem indicada, evitaria a ocorrência de reações adversas e promoveria a melhora da qualidade de vida dos idosos (MALAQUIAS et al., 2016). Fica evidente a necessidade de implementação da promoção do uso racional de medicamentos pelos profissionais de saúde, não só pra proteger a saúde dos pacientes, mas também para educar a população, reduzindo assim os riscos relacionados ao uso de fármacos sem orientação (OLIVEIRA, et al., 2018).

Quando contestados sobre o tempo que fazem uso destes medicamentos, $31 \%$ dos idosos disseram fazer uso de 1 mês a 5 anos; $26,8 \%$ de 6 a 10 anos; $29,6 \%$ de 11 a 20 anos; e 12,6\% acima de 20 anos. Um ponto importante a salientar, é que 18,3\% dos participantes da pesquisa não sabiam qual a função dos medicamentos que consumia, mesmo, em média, fazendo uso do fármaco a mais de 12 anos.

A orientação sobre a ação dos medicamentos, a forma do tratamento e as dúvidas sobre a enfermidade devem ser esclarecidas aos idosos devido a sua proximidade com os fármacos que vão ser utilizados geralmente, de forma diária. Assim, a atenção farmacêutica torna-se fundamental para melhoria da manutenção do estado de saúde destes pacientes (BORTOLON, et al., 2007).

A bula que acompanha os medicamentos é um documento legal sanitário, que fornece informações e orientações necessárias sobre os fármacos, para seu uso seguro e tratamento eficaz. Quando indagados sobre o entendimento da bula, 22,5\% dos idosos entrevistados relataram não entender as informações contidas neste documento, e 20\% afirmaram que sequer liam o material. Os dados encontrados na atual pesquisa são preocupantes, pois autores afirmaram que no Brasil, a bula é o principal material informativo fornecido aos pacientes pela indústria farmacêutica (MEROLA; EL-KHATIB; GRANJEIRO, 2005).

Em estudo realizado por Merola; El-Khatib; Granjeiro (2005), os pesquisadores constataram que $80 \%$ dos idosos não sabiam interpretar a bula, ou seja, não entendiam as indicações necessárias para o uso do medicamento. Desta forma, a atuação do farmacêutico novamente deve ser destacada, pois este profissional conhece todos os aspectos relacionados aos fármacos e pode então, facilmente, instruir o usuário quanto a utilização de forma correta e segura (BARBOSA et al., 2018).

O uso de medicamentos sem prescrição médica é contraindicado. Quando contestados sobre a prescrição dos medicamentos de uso contínuo, 98,6\% dos entrevistados disseram ter sido prescritos por médico, porém, 1,4\% afirmaram que não 
houve prescrição médica e que faz uso por indicação de parentes. Este fato é bastante preocupante devido a possibilidade de intoxicação farmacológica, ou ocorrência de interações medicamentosas indesejadas.

Além disso, 89,5\% dos idosos relataram fazer uso da automedicação quando estão com sintomas, como dores em geral e febre, citando o uso principalmente de paracetamol, dipirona, e medicamentos compostos por associações como, Citrato de Orfenadrina, Dipirona Sódica e Cafeína Anidra.

A receita médica é dispensada para aquisição de algumas substâncias, porém o uso de forma inadequada destes fármacos pode causar sérios prejuízos aos usuários, principalmente pelo princípio de que estas substâncias são inócuas ao organismo (CHEHUEN NETO et al., 2006).

Quando perguntados sobre receber auxilio quando vão ingerir os fármacos, relacionado a horário, dose e função, 85,9\% disseram não receber, ou seja, fazem uso sozinhos, e $88,7 \%$ relataram nunca ter se confundido, porém, $11,3 \%$ já se confundiu. $A$ confusão relatada foi com relação a inversão de horários, ingestão duplicada de medicamento, e erros devido a semelhança das drágeas. Este dado é muito importante devido ao risco de superdosagem, intoxicação medicamentosa, ocorrência de efeitos colaterais e também a falta de efeito esperado da medicação quando a mesma é esquecida ou substituída por outro com drágea e/ou embalagem semelhante.

A instrução de uso de qualquer fármaco deve ser feita pelo farmacêutico no momento de sua aquisição. Na pesquisa, $12,5 \%$ dos idosos afirmaram não receber instruções com relação ao uso dos medicamentos.

A orientação aos idosos referente ao uso dos medicamentos prescritos deve ser feita pelo farmacêutico e compreende uma importante ação de atenção farmacêutica. Estas orientações podem ser realizadas por vários meios, por exemplo, para pacientes analfabetos, fazer desenhos (sol e lua) indicando o horário do esquema posológico prescrito; usar símbolos ou etiquetas coloridas em caixas semelhantes ou medicamentos com drágeas que possam ser confundidas; orientar sobre práticas de exercícios físicos e boa alimentação que irão auxiliar no bom resultado do tratamento; e sempre instruir ao não uso de medicamentos sem prescrição médica (VIEIRA; HOSSNE, 2015).

Referente a atenção farmacêutica, $47,5 \%$ dos idosos disseram não saber do que se trata. Este resultado pode indicar a falta de empenho relacionado ao tema pelos profissionais da saúde, pois dentro deste sistema, o farmacêutico representa uma das 
últimas oportunidades em identificar, corrigir ou reduzir os riscos possíveis associados ao uso de terapêutica inadequada (SILVA et al., 2018).

Geralmente, os pacientes enxergam apenas o médico como um profissional habilitado para acompanhamento do tratamento pós instituição do uso de fármacos (MEROLA; EL-KHATIB; GRANJEIRO, 2005). Por isso, a intervenção farmacêutica deve ser realizada para mudança desta visão com objetivo de solucionar ou prevenir falhas nas prescrições, pois os cuidados farmacêuticos melhoram os resultados clínicos e econômicos dos tratamentos (ARAUJO et al., 2017).

De acordo com $98,8 \%$ dos idosos entrevistados, o auxílio prestado pelo farmacêutico no momento da compra do medicamento com relação a como usá-lo e o acompanhamento do uso é muito importante. Destes, 92,5\% gostariam de contar com a ajuda de um farmacêutico para realizar o uso de seus medicamentos.

A ajuda profissional farmacêutica é uma atribuição no âmbito de atenção à saúde. Assim, a obtenção de dados sobre os fármacos e sobre a saúde do paciente são funções também destinadas a estes profissionais. A elaboração de um plano de atenção farmacêutica que compreende educação, orientação, intervenções e parâmetros farmacêuticos acrescenta valores a terapia medicamentosa atribuída e consequentemente aprimora a atenção à saúde (SANTOS et al, 2016).

Este conjunto de ações do farmacêutico vem crescendo e se destacando entre a população idosa, e é utilizado como um pilar das estratégias de atenção à saúde, pois atua na melhoria da qualidade de vida do paciente prevenindo a reincidência de enfermidades e atuando na correta farmacoterapia (LIMA et al., 2016).

\section{CONCLUSÄO}

Esta pesquisa permitiu concluir que a maioria dos idosos que frequentavam o $\mathrm{CCl}$ eram mulheres, com faixa etária de idosos jovens, e que utilizam medicamentos de uso contínuo, entre 2 a 4 medicamentos diários, sendo a maioria anti-hipertensivos e diuréticos.

Praticamente a metade dos idosos (47,5\%) desconhecem a atenção farmacêutica e a maioria utiliza medicamentos diariamente sem contar com auxílio farmacêutico para o uso, o que pode causar problemas relacionados a reações adversas, interações medicamentosas e o uso indiscriminado de alguns fármacos.

Para os idosos, a atenção farmacêutica é fundamental, pois a idade avançada contribui para erro em autoadministrações, devido a quantidade excessiva de 
medicamentos ingeridos diariamente, alterações na visão, destreza manual e cognitivas. $\mathrm{O}$ acompanhamento do farmacêutico ao paciente permite a promoção de educação em saúde, a isenção dos riscos de erros de administração de fármacos, a melhoria do quadro clínico e qualidade de vida do idoso, além de seu reconhecimento profissional.

Mesmo com todas as vantagens relacionadas a aplicação da atenção farmacêutica, esta prática não é realizada na rotina dos pacientes, como detectado neste trabalho, o que reforça a importância de pesquisas nesta área, pois as atitudes do farmacêutico neste campo de atuação são inúmeras, e faz com que este profissional seja cada vez mais requisitado, devido ao envelhecimento populacional, aumento de expectativa de vida e aumento da procura pelos serviços de saúde.

Indica-se desta forma a realização de atenção farmacêutica por meio de ações educativas e orientações sobre o regime terapêutico, pois estas ações promovem benefícios à saúde do paciente e ao processo de promoção da saúde. O objetivo desta não é intervir no diagnóstico ou na prescrição de medicamentos e nem nas atribuições do médico, mas sim garantir uma farmacoterapia racional, segura e custo-efetiva.

\section{REFERÊNCIAS}

AGUIAR, P. et al. Avaliação da farmacoterapia de idosos residentes em instituições asilares no nordeste do Brasil. Latin Americam Journal of Pharmacy, v.27, n.3, p.454-459, 2008.

ANDRADE, M. A.; SILVA, M. V. S.; FREITAS, O. Assistência Farmacêutica como Estratégia para o Uso Racional de Medicamentos em Idosos. Semina: Ciências Biológicas e da Saúde, v.25, n.1, p.56-60, 2004.

ARAUJO, E. O. et al. Intervenções farmacêuticas em uma unidade de terapia intensiva de um hospital universitário. Revista Brasileira de Farmácia Hospitalar e Serviços de Saúde, v.8, n.3, p.25-30, 2017.

BARBOSA, M.; NERILO, S. B. Atenção farmacêutica como promotora do uso racional de medicamentos. Revista UNINGA Review, v. 30, n. 2, jan. 2018.

BATISTA, N. N. L. A. L.; VIEIRA, D. J. N.; SILVA, G. M. P. Caracterização de idosos participantes de atividade física em um centro de convivência de Teresina-PI. Enfermagem em Foco, v.3, n.1, p.7-11, 2012.

BORTOLON, P. C.; KARNIKOWSKI, M. G.; ASSIS, M. Automedicação versus indicação farmacêutica: o profissional de farmácia na atenção primária à saúde do idoso. Revista APS, v.10, n.2, p. 200-209, 2007.

BURTON, D. et al. Bridging the gap: ageing, pharmacokinetics and pharmacodynamics. Journal Pharmaceutical Pharmacology, v.57, p.671-679, 2005. 
CADOGAN, C.; RYAN, C.; HUGHES, C. Appropriate polypharmacy and medicine safety: when many is not too many. Journal Drug Safety, v. 39, n. 2, p.109-16, 2016.

CAZARIM, M.; ARAÚJO, A. O paciente idoso sob o aspecto da utilização de antimicrobianos: repercussão ao sistema público de saúde brasileiro (SUS). Revista de Ciências Farmacêuticas Básica e Aplicada, v. 32, n. 3, p. 305-311, 2012.

CHEHUEN NETO, J. et al. Automedicação entre Estudantes da Faculdade de Medicina da Universidade Federal de Juiz de Fora. HU Revista, v.32, n.3, p.59-64, 2006.

CONCEIÇÃO, S. B. et al. Envelhecimento populacional com foco no uso racional de medicamentos: o papel do farmacêutico. Revista Intersaúde, v.1, n.1, p. 37-46, 2019.

COSTA, S. T. S.; ALVES, F. C. E. F.; JESUS, M. R. Auxiliar farmacêutico na saúde do idoso quanto ao uso do medicamento controlado. GETEC, v.8, n.21, p.12-22, 2019.

FREIRE, G. V. et al. Perfil de idosos que frequentam um centro de convivência da terceira idade. Revista Interdisciplinar, v. 8, n. 2, p. 11-19, 2015.

FREITAS, M. S.; TOMA, W. Atenção Farmacêutica a Pacientes Atendidos na Clínica de Implantodontia da Universidade Santa Cecília. In: ENCONTRO NACIONAL DE PÓSGRADUAÇÃO, 3, 2019, São Paul. Anais... São Paulo: UNISANTA, 2019, p.263-266.

IVAMA, A. M. et al. Atenção farmacêutica no Brasil: trilhando caminhos: relatório 20012002. Brasília DF: Organização Pan-Americana da Saúde, 2002. p. 46.

LIMA, T. A. M. et al. Acompanhamento farmacoterapêutico em idosos. Revista Arquivos de Ciências da Saúde, v. 23, n. 1, p. 52-57, 2016.

LOYOLA FILHO, A.; UCHOA, E.; LIMA-COSTA, M. F. Estudo epidemiológico de base populacional sobre uso de medicamentos entre idosos na Região Metropolitana de Belo Horizonte, Minas Gerais, Brasil. Cadernos de Saúde Pública, v.22, n.12, p.2657-2667, 2006.

LUPPI, G.; CARVALHO, M. F. C. Atenção farmacêutica em pacientes geriátricos: uma experiência no Centro de Referência do Idoso. Cadernos da Faculdades Integradas São Camilo, v.11, n.4, p. 90-96, 2005.

MALAQUIAS, B. et al. Avaliação das prescrições de medicamentos a idosos em um ambulatório de geriatria. Medicina (Ribeirão Preto), v. 49, n. 5, p. 440-50, 2016.

MANSO, M. E. G.; BIFFI, E. C. A.; GERARDI, T. J. Prescrição inadequada de medicamentos a idosos portadores de doenças crônicas em um plano de saúde no município de São Paulo, Brasil. Revista Brasileira de Geriatria e Gerontololgia, v.18, n.1, pp.151-164, 2015.

MARIN, M. J. S. et al. Caracterização do uso de medicamentos entre idosos de uma unidade do Programa Saúde da Família. Cadernos de Saúde Pública, v. 24, p. 1545-1555, 2008.

MENESES, A. L. L.; SÁ, M. L. B. Atenção farmacêutica ao idoso: fundamentos e propostas. Geriatria \& Gerontologia, v.4, n.3, p.154-161, 2010. 
MEROLA, Y. L.; EL-KHATIB, S.; GRANJEIRO, P. A. Atenção farmacêutica como instrumento de ensino. Infarma, v.17, n.7/9, p. 70-72, 2005.

NICODEMO, D.; GODOI, M. P. Juventude dos anos 60-70 e envelhecimento: estudo de casos sobre feminização e direitos de mulheres idosas. Revista Ciência em Extensão, v.6, n.1, p.40-53, 2010.

NOBREGA, O. T.; KARNIKOWSKI, M. G. A terapia medicamentosa no idoso: cuidados na medicação. Ciência e Saúde Coletiva, v.10, n.2, p.309-313, 2005.

NOVAES, M. R. C. G. Assistência farmacêutica ao idoso - uma abordagem multiprofissional.1ed. Brasília: Thesaurus, 2007.

OLIVEIRA, H. S. B.; CORRADI, M. L. G. Aspectos farmacológicos do idoso: uma revisão integrativa de literatura. Revista de Medicina, São Paulo, v. 97, n. 2, p. 165-176, 2018.

PARRINI, K. C. M. S.; PINHEIRO, M. E. N.; SILVA, T. B. Implementação da atenção farmacêutica e farmacoterapia na terceira idade em abrigos, asilos e casa de repouso na cidade de Teresópolis e palestras sobre o uso correto de medicamentos. Revista da JOPIC, v.2, n.4, p.11-14, 2019.

ROMANO-LIEBER, N. S. et al. Revisão dos estudos de intervenção do farmacêutico no uso de medicamentos por pacientes idosos. Cadernos de Saúde Pública, v.18, n.6, 14991507, 2002.

SALDANHA, A. L.; CALDAS, C. P. Saúde do idoso: a arte de cuidar. 2ed. Rio de Janeiro: Interciência, 2004.

SANTOS, S. L. F. et al. Serviço de atendimento farmacêutico ao idoso: relato de experiência de educação em saúde. Centro de ciências da saúde Santa Maria, v. 42, n. 2, p. 225231, 2016.

SCHRODER, T. et al. Atenção farmacêutica à paciente idosa que faz uso de múltiplos medicamentos. In: 6ำ CONGRESSO INTERNACIONAL EM SAÚDE, 2019. Anais...ljuí: UNIJUI, 2019.

SILVA, A. L.; NASCIMENTO, R.; GRASSI, L. T. Atenção farmacêutica ao idoso. Revista Saberes da FAPAN, v.3, n.1, p.39-49, 2016.

SILVA, A. C. S. et al. Acompanhamento farmacoterapêutico em unidade de terapia intensiva respiratória: descrição e análise de resultados. Einsten, v. 16, n. 2, p.1-7, 2018.

SILVA, R. Caracterização da população idosa usuária dos serviços do centro de referência especializado de assistência social (CREAS) de Mariana-Minas Gerais. 2019. 72f. Trabalho de conclusão de curso (Serviço Social) - Universidade Federal de Ouro Preto, Mariana. 2019.

SOUZA NETO, J. C. et al. Perfil de idosos frequentadores de um Centro de Convivência Público de Teresina-PI. Jornal Interdisciplinar de Biociências, v.3, n.2, p. 13-18, 2018.

VIEIRA, S.; HOSSNE, W. S. Metodologia científica para a área da saúde. Brasil: Elsevier, 2015. 